\title{
Metric Lagrangians with two propagating degrees of freedom
}

\author{
Kirill Krasnov \\ School of Mathematical Sciences, University of Nottingham, Nottingham, NG7 2RD, UK
}

(Dated: October 21, 2009)

\begin{abstract}
There exists a large class of generally covariant metric Lagrangians that contain only local terms and describe two propagating degrees of freedom. Trivial examples can be be obtained by applying a local field redefinition to the Lagrangian of general relativity, but we show that the class of two propagating degrees of freedom Lagrangians is much larger. Thus, we exhibit a large family of non-local field redefinitions that map the Einstein-Hilbert Lagrangian into ones containing only local terms. These redefinitions have origin in the topological shift symmetry of BF theory, to which GR is related in Plebański formulation, and can be computed order by order as expansions in powers of the Riemann curvature. At its lowest non-trivial order such a field redefinition produces the (Riemann $)^{3}$ invariant that arises as the two-loop quantum gravity counterterm. Possible implications for quantum gravity are discussed.
\end{abstract}

PACS numbers: 04.60.-m

Loop divergences in quantum gravity require higher derivative counterterms to be added to the Lagrangian [1], 2]. Such higher derivative terms typically introduce new propagating degrees of freedom (DOF) that generally lead to instabilities, see [3] for an emphasis of this point. The only known way to avoid these instabilities is to have a well-behaved underlying theory describing the new DOF, for example string theory. The higher-derivative metric Lagrangian is then an effective one obtained by integrating out some underlying nongravitational DOF. In this letter we show that there exists a potentially attractive alternative: higher derivative counterterms can be added to the gravitational Lagrangian without adding new degrees of freedom.

Field redefinitions play an important role in our construction, so we start by briefly recalling some relevant facts. Quantum gravity, with its negative mass dimension coupling constant, is non-renormalizable in the sense that an infinite number of counterterms is required to absorb all arising divergences. However, while in a typical renormalizable theory transformations that absorb infinities are limited to field and coupling constant multiplicative renormalizations, the field redefinition freedom available in a theory with a dimensionful coupling is considerably larger. Thus, in the case of (pure, i.e with no matter couplings) quantum gravity one can perform field redefinitions of the schematic type $h \rightarrow h+\sum_{n} G^{n} \partial^{2 n} h+\ldots$, where $h$ is the graviton field, $G$ is Newton's constant, and dots denote terms of higher order in the metric perturbation. The power of $G$ here is as relevant for the case of 4 spacetime dimensions, but similar field redefinitions, with an appropriate modification of the power of $G$ are available in other dimensions as well. Such field redefinitions, being local, are known not to change the S-matrix of the theory, see e. g. [4], section 2, as well as [5], section 10 for a discussion of this point. The availability of these field redefinitions implies that many of the arising counterterms are unphysical in the sense that they can be disposed off without any effect on the S-matrix. An extreme example of this situation arises when, in spite of divergences being present, they can all be removed by local field redefinitions without affecting the S-matrix. In this case one says that the theory is (on-shell) finite. An example of a finite but power-counting non-renormalizable theory is given by pure quantum gravity in 3 spacetime dimensions.

For later purposes we note that classically a local metric field redefinition maps the Einstein-Hilbert Lagrangian into a complicated metric Lagrangian containing an infinite number of local terms. The new Lagrangian, however, still describes just two propagating DOF. This can be seen by following the Ostrogradski method of introducing new variables for higher time derivatives. One then observes that the arising Lagrangian is highly degenerate and generates many constraints that remove all but the DOF of the original system. It is simplest to see this mechanism at work by considering a higher derivative field redefinition applied to a finite-dimensional dynamical system.

A celebrated result of [1] is that one-loop divergences of pure quantum gravity in 4 spacetime dimensions can be removed by a local field redefinition and so the theory is one-loop finite. It was for some time hoped that the finiteness may persist to all loops, but an explicit twoloop computation [2] showed that the term (Riemann $)^{3}$ that is not removable by a local field redefinition is needed to absorb the divergences arising.

On the other hand, non-local redefinitions, i.e. involving negative powers of $\square=\partial_{\mu} \partial^{\mu}$, generically do change the S-matrix. Still, an appropriate ghost action can be introduced to offset their effect, see [5]. However, such field redefinitions typically map a local action to a nonlocal one, and are thus uninteresting for the purpose of eliminating local counterterms. Indeed, the simplest example is given by the free field Lagrangian $(1 / 2)\left(\partial_{\mu} \phi\right)^{2}$, which, after a redefinition $\phi \rightarrow \phi+(\mathcal{O} / \square) \phi$, where $\mathcal{O}$ is some local operator, goes into a non-local Lagrangian containing $1 / \square$. 
We now show that in the case of gravity (in 4 spacetime dimensions) the class of field transformations that map a local Lagrangian into again a local one is much larger than that consisting of local field redefinitions. In other words, there exists an (infinite-parameter) family of non-local field redefinitions that map the EinsteinHilbert Lagrangian into a generally covariant metric Lagrangian containing only local terms. The redefinition can be computed order by order in perturbation theory as follows. At lowest order, it is the local transformation

$$
h_{\mu \nu} \rightarrow h_{\mu \nu}+\alpha R_{\mu \nu}+\beta \eta_{\mu \nu} R
$$

that produces $R^{\mu \nu} R_{\mu \nu}$ and $R^{2}$ invariants. Here $\eta_{\mu \nu}$ is the Minkowski metric, and $R_{\mu \nu}, R$ are the Ricci tensor and scalar for the perturbation $h_{\mu \nu}$ respectively. At the next order our field redefinition produces the $(\text { Riemann })^{3}$ invariant as well as other on-shell vanishing ones and is given by

$$
h_{\mu \nu} \rightarrow h_{\mu \nu}+\frac{\gamma}{\square} \partial^{\alpha} \partial^{\beta} R_{\mu \alpha}^{\gamma \delta} R_{\nu \beta \gamma \delta},
$$

plus a set of local terms. The reason why (2) produces

$$
\frac{\gamma}{4} \int R_{\mu \nu}{ }^{\rho \sigma} R_{\rho \sigma}^{\alpha \beta} R_{\alpha \beta}{ }^{\mu \nu}
$$

is that this quantity can be written as:

$$
\int \mathcal{E}^{\mu \nu} \frac{\gamma}{\square} \partial^{\alpha} \partial^{\beta}\left(R_{\mu \alpha}^{\gamma \delta} R_{\nu \beta \gamma \delta}-\frac{1}{2} \eta_{\mu \nu} R_{\alpha}{ }^{\rho \gamma \delta} R_{\beta \rho \gamma \delta}\right),
$$

where $\mathcal{E}_{\mu \nu}=R_{\mu \nu}-\frac{1}{2} \eta_{\mu \nu} R$. This is checked using the easily verifiable identity

$$
\partial_{[\alpha} \partial^{[\beta} R_{\mu]}^{\nu]}=\frac{1}{4} \square R_{\alpha \mu}^{\beta \nu}
$$

that holds to first order in the perturbation field. Note that the reason why the last term in brackets in (4) was not included in (2) is that it is proportional to $\eta_{\alpha \beta}$, and thus gives rise to a local term.

The structure of the field redefinition at higher orders is similar to (2) in that the non-local operator $\partial^{\alpha} \partial^{\beta} / \square$ is applied to a rank 4 tensor constructed from an appropriate power of the Riemann curvature tensor (and its covariant derivatives), plus a set of local terms. Importantly, at higher orders there are also terms containing higher negative powers of $\square$. These are needed to eliminate terms arising as powers of lower order non-localities. The above prescription can be carried out order by order, but this becomes technically difficult at higher orders. Below we present an alternative description of the same field redefinition that guarantees that it can be extended to any order and gives an algorithmic procedure for computing it.

At every order the non-local field redefinition sketched introduces a set of parameters that, after it is applied to the Einstein-Hilbert Lagrangian, translate into parameters of the arising local metric Lagrangian. When truncated to any given order, the Lagrangian one obtains contains many new DOF stemming from its higherderivative nature. However, the complete Lagrangian with its infinite number of local terms describes just two propagating DOF. To see this we must introduce a different and at first unrelated description of this class of Lagrangians.

An alternative description of the two propagating DOF metric Lagrangians is provided by an infinite-parameter family of deformations of general relativity first described in [6], building upon works [7, 8, 2, 10]. One starts with an observation [7] that (complexified) Einstein's general relativity can be rewritten as a generally-covariant theory of an $\mathrm{SO}(3, \mathbb{C})$ connection. This suggests generalizations, leading to an infinite-parameter family [6] of theories describing two propagating degrees of freedom (DOF) and containing GR. These two propagating DOF gravity theories can be rewritten in metric terms and can be shown to be obtainable from GR precisely by the above nonlocal field redefinitions.

These deformations of GR can be described compactly as follows. Let $A^{i}, i=1,2,3$ be an $\mathrm{SO}(3, \mathbb{C})$ connection and $F^{i}=d A^{i}+(1 / 2) \epsilon^{i j k} A^{j} \wedge A^{k}$ be its curvature two-form. The action of the theory is just the most general generally-covariant action that can be constructed for $A^{i}$. Thus, consider the 4-form $F^{i} \wedge F^{j}$. Choosing an arbitrary volume 4 -form ( $v o l)$ we can write $F^{i} \wedge F^{j}=(v o l) \Omega^{i j}$, with $\Omega^{i j}$ being defined only modulo rescalings $(\mathrm{vol}) \rightarrow \alpha(\mathrm{vol}), \Omega^{i j} \rightarrow(1 / \alpha) \Omega^{i j}$. Introduce a scalar-valued function $f(X)$ of $3 \times 3$ symmetric matrices $X^{i j}$ that is $\mathrm{SO}(3, \mathbb{C})$-invariant $f\left(O X O^{T}\right)=$ $f(X), O \in \mathrm{SO}(3, \mathbb{C})$, holomorphic, and homogeneous of degree one $f(\alpha X)=\alpha f(X)$. This function can be applied to the 4-form $F^{i} \wedge F^{j}$ with the result being a 4-form $f\left(F^{i} \wedge F^{j}\right)=(v o l) f(\Omega)$, independent of which volume 4form is used. Thus, we can write a generally-covariant and gauge-invariant action as follows:

$$
S[A]=\int f\left(F^{i} \wedge F^{j}\right) .
$$

It can then be shown that for a generic $f(\cdot)$ this gives a theory that describes 2 (complex) propagating DOF. This can be seen by noting that the phase space of this theory is parametrized by pairs (spatial projection of the connection, conjugate momentum). The theory is diffeomorphism and gauge-invariant which means that there are $4+3$ first-class constraints acting on the phase space. With the configuration space being $3 \times 3$ dimensional, this leaves 2 physical DOF. It can also be shown, see [7], that general relativity belongs to the class (6) with the function $f(\cdot)$ being the $\delta$-function projecting onto:

$$
\operatorname{Tr} \Omega^{2}=\frac{1}{2}(\operatorname{Tr} \Omega)^{2} .
$$


Note that the clause about $f(\cdot)$ being generic is important, for the Lagrangian $\operatorname{Tr} F \wedge F$, which is also in the class (6), is a total divergence and corresponds to a theory without propagating DOF. The description of the theory given here is new, but can be shown to be equivalent to one given in 6].

As was realized in [11, 12], the theory (6) can be put into a form that makes the spacetime metric it describes more explicit. In the retrospect, this is done via a standard "duality" trick of introducing a set of new fields that are later taken to be fundamental, with the fields of the original formulation to be integrated out. The new fields in our case are two-form fields $B^{i}$ that are valued in the Lie-algebra of $\mathrm{SO}(3, \mathbb{C})$. The new action is given by:

$$
S[B, A]=\int B^{i} \wedge F^{i}-\frac{1}{2} V\left(B^{i} \wedge B^{j}\right) .
$$

Here $V(\cdot)$ is again a holomorphic, $\mathrm{SO}(3, \mathbb{C})$-invariant, homogeneous function of order one so that it can be applied to the 4-form $B^{i} \wedge B^{j}$. Integrating the two-form field $B^{i}$ out by solving its (algebraic) field equations one gets back (6) with $f(\cdot)$ being an appropriate Legendre transform of $V(\cdot)$. One can now take the two-form field $B^{i}$ to be fundamental, and eliminate $A^{i}$ completely by solving its field equations that are algebraic. This converts (8) into a second-order theory for the two-form field $B^{i}$.

The spacetime metric described by the theory becomes almost manifest in the two-form field formulation (8). Thus, it can be shown that the theory is about the spacetime (conformal) metric with respect to which the set of two-forms $F^{i}$ (or, equivalently, $B^{i}$ ) is self-dual. It is not hard to show that there is a unique such (conformal) metric, see e.g. [13]. Explicitly, this metric is given by:

$$
\sqrt{-g} g_{\mu \nu} \sim \epsilon^{i j k} B_{\mu \alpha}^{i} B_{\nu \beta}^{j} B_{\rho \sigma}^{k} \tilde{\epsilon}^{\alpha \beta \rho \sigma} .
$$

Introducing the conformal metric (9), the action (8) can be explicitly rewritten in a second-order form as that of the metric plus a set of auxiliary non-propagating fields. This is done by introducing a set of special self-dual "metric" two-forms $\Sigma^{i}$ that satisfy:

$$
\Sigma^{i} \wedge \Sigma^{j} \sim \delta^{i j} .
$$

These forms are easily constructed by introducing a tetrad $\theta^{I}, I=(0, i)$ for the metric, and taking the selfdual part of the two-form $\theta^{I} \wedge \theta^{J}$ given by:

$$
\Sigma^{i}=\mathrm{i} \theta^{0} \wedge \theta^{i}-\frac{1}{2} \epsilon^{i j k} \theta^{j} \wedge \theta^{k} .
$$

It can be shown that the knowledge of two-forms that are self-dual and satisfy (10) is equivalent to the knowledge of the metric. A general self-dual two-form $B^{i}$ can then be written as:

$$
B^{i}=b_{j}^{i} \Sigma^{j}
$$

where $b_{j}^{i}$ are arbitrary scalars. The theory (8) with the connection $A^{i}$ eliminated via its field equations then becomes a second-order theory of the metric described by $\Sigma^{i}$ and the non-propagating scalars $b_{j}^{i}$. A simple phase space analysis shows that the theory contains only two propagating DOF. The scalars can then be integrated out to produce a purely metric theory. This leads to a Lagrangian given by an infinite expansion in terms of local curvature invariants, which describes two propagating DOF by construction. The above discussion was phrased in terms of complex spacetime metrics, but appropriate reality conditions can be imposed, and the story repeats itself for real Lorentzian signature metrics.

Thus, we have seen that among all generally-covariant local (i.e. containing only local terms) metric Lagrangians there is an infinite-parameter subset that describes, as GR, only two propagating DOF. To see the non-local field redefinitions that relate these Lagrangians to GR we note that in the formulation (8) the first BF term possesses a large symmetry $B^{i} \rightarrow B^{i}+D \eta^{i}$, where $\eta^{i}$ is a Lie-algebra valued one-form, and $D$ is the covariant derivative with respect to the connection $A^{i}$. A subgroup of this symmetry group is formed by spacetime diffeomorphisms. The second, potential term of the action is only invariant under this diffeomorphism subgroup, and this is the reason why the above "topological shift" transformation is not a symmetry of the whole action. This is also the reason why (8), unlike BF-theory, has propagating DOF.

The topological shift transformation described can be used to map (8) to the Einstein-Hilbert action plus a simple potential term for a set of scalars that are decoupled from the metric. At the linearized level this was noted in [14]. To see this for the full theory, we use the observation of [15] that the Einstein-Hilbert Lagrangian can be written in BF form in terms of the two-forms $\Sigma^{i}$ constructed from the metric. We then note that the topological shift symmetry can be used to transform any two-form field $B^{i}$ into a "metric" one $\Sigma^{i}=B^{i}+D \eta^{i}$ satisfying (10). A detailed demonstration of this fact is beyond the scope of this letter, but it is not hard to see that the number of parameters available in the oneform field $\eta^{i}$, modulo diffeomorphisms and modulo the "gauge" $\eta^{i} \rightarrow \eta^{i}+D \phi^{i}$, where $\phi^{i}$ is a Lie-algebra valued zero-form, matches precisely the number of "metricity" equations (10) to be satisfied. It can also be shown, at least perturbatively around the Minkowski background, that the two-form $\Sigma^{i}$ arising this way is unique. This discussion shows that the first BF-term of the action (8) can be written as the Einstein-Hilbert one for the metric obtained from $B^{i}$ by the topological shift symmetry. The second term in (8) then becomes a potential for the non-propagating scalars contained in $B^{i}$. By their field equations these scalars are set to a value corresponding to a minimum of the potential, and decouple, which leaves one with the Einstein-Hilbert action (with a cosmological 
constant whose value is given by the minimum of $V(\cdot)$ ) for the metric described by $\Sigma^{i}$. This shows that there exists a field redefinition that maps (8) into the EinsteinHilbert action. The field redefinition in question involves solving a differential equation for the shift one-form parameter $\eta^{i}$, and is thus non-local. It can be computed order by order perturbatively expanding the metric(s) around the Minkowski background. Details will appear elsewhere. The end result is given by the transformation that was described in the beginning of this letter, with parameters of the transformation related to those of the potential $V(\cdot)$.

To summarize, we have seen that the set of generallycovariant local metric Lagrangians describing two propagating DOF is larger than the one obtainable from GR by local field redefinitions, and admits a very compact description (6). It is obtainable from GR by special nonlocal field redefinitions that stem from the topological shift symmetry of the BF-part of the action (8).

Let us conclude by discussing what the existence of an (infinitely) large class of two propagating DOF metric Lagrangians may imply for the problem of quantum gravity. The fact that the (Riemann $)^{3}$ counterterm needed at two loops is contained in our two propagating DOF Lagrangians suggests that it may be possible to device a renormalization scheme for gravity so that the counterterm-corrected Lagrangian remains within two DOF class at every order of perturbative expansion. For this to be possible the class of theories (6) must be closed under renormalization, which appears plausible, since the Lagrangian in (6) is just the most general one compatible with gauge and diffeomorphism invariance. Such a renormalization scheme, if possible, would give a quantum theory of gravity with two propagating DOF, which would be in striking contrast with other quantum gravity scenarios (e.g. string theory) that typically introduce new DOF.

If this scenario was possible, one would face a question about implications of the non-local topological shift symmetry described. While generically non-local field redefinitions do change the S-matrix, our redefinitions are certainly of a very special nature since a local action is mapped again into a local one. Therefore, the general conclusion has to be carefully re-examined. Preliminary considerations suggest that our non-local transformations might not affect the S-matrix. If this was so, then all quantum divergences were disposable without affecting the S-matrix, and the quantum theory would be finite. It is of considerable interest to see if this vision can be realized.

The story described is that for pure, i.e. not coupled to any matter sources, gravity. Indeed, coupling to usual type matter essentially removes the field redefinition freedom. However, similarly to how the one-loop finiteness result [1] extends to special matter couplings provided by supergravity theories, our story may also be applicable to gravity coupled to at least certain types of matter. This will be described elsewhere.

This work was supported by an EPSRC Advanced Fellowship. The author is grateful to Alexey Boyarsky for important suggestions on the draft, and to Yuri Shtanov for many discussions on the subject.

[1] G. 't Hooft and M. J. G. Veltman, Annales Poincare Phys. Theor. A 20, 69 (1974).

[2] M. H. Goroff and A. Sagnotti, Nucl. Phys. B 266, 709 (1986).

[3] R. P. Woodard, arXiv:0907.4238 [gr-qc].

[4] N. Marcus and A. Sagnotti, Nucl. Phys. B 256, 77 (1985).

[5] G. 't Hooft and M. J. G. Veltman, NATO Adv. Study Inst. Ser. B Phys. 4, 177 (1974).

[6] I. Bengtsson, Phys. Lett. B 254, 55 (1991).

[7] R. Capovilla, T. Jacobson and J. Dell, Phys. Rev. Lett. 63, 2325 (1989).

[8] R. Capovilla, T. Jacobson and J. Dell, Class. Quant. Grav. 8 (1991) 59.

[9] R. Capovilla, Nucl. Phys. B 373, 233 (1992).

[10] I. Bengtsson and P. Peldan, Int. J. Mod. Phys. A 7, 1287 (1992)

[11] K. Krasnov, arXiv:hep-th/0611182

[12] K. Krasnov, Class. Quant. Grav. 26, 055002 (2009) arXiv:0811.3147 [gr-qc]].

[13] H. Urbantke, J. Math. Phys. 25, 2321 (1984).

[14] L. Freidel, arXiv:0812.3200 [gr-qc].

[15] J. F. Plebanski, J. Math. Phys. 18, 2511 (1977). 